\title{
氧化镍/镍/碳微球原位制备及葡萄糖传感性能
}

\author{
秦冬昱 ${ }^{1,2}$, 何晓龙 ${ }^{1}$, 聂秋林 ${ }^{1}$, 殷好勇 ${ }^{1}$, 袁求理 ${ }^{2}$ \\ (杭州电子科技大学 1. 材料与环境工程学院; 2. 理学院, 杭州 310018)
}

摘 要: 以葡萄糖、六水合氯化镍和尿素为原料, 通过水热反应一步制备前驱体 $\mathrm{Ni}(\mathrm{OH})_{2} / \mathrm{C}$, 在高纯度氮气中煅烧 获得 $\mathrm{NiO} / \mathrm{Ni} / \mathrm{C}$ 微球三元复合材料。采用扫描电镜 $(\mathrm{SEM}) 、$ 透射电镜(TEM)、X 射线衍射 $(X R D) 、 X$ 射线光谱(EDS) 和拉曼(Raman)等手段, 分析 $\mathrm{NiO} / \mathrm{Ni} / \mathrm{C}$ 微球三元复合材料的形貌结构以及物相组成。结果表明: $\mathrm{NiO} / \mathrm{Ni} / \mathrm{C}$ 微球为 珊瑚花状结构, 直径约 $1.7 \mu \mathrm{m}, \mathrm{Ni} 、 \mathrm{NiO}$ 呈立方相。通过循环伏安法和计时电流法研究了 $\mathrm{NiO} / \mathrm{Ni} / \mathrm{C}$ 微球三元复 合材料的电化学行为及葡萄糖传感性能。当 $\mathrm{Ni} / \mathrm{NiO}$ 摩尔比为 0.19 时, 形成的 $\mathrm{NiO} / \mathrm{Ni} / \mathrm{C}$ 三元复合微球具有优异 的葡萄糖传感性能，其灵敏度为 $241.09 \mu \mathrm{A} \cdot \mathrm{mmol} /\left(\mathrm{L} \cdot \mathrm{cm}^{2}\right)$, 线性响应范围为 $10 \mu \mathrm{mol} / \mathrm{L} \sim 5.05 \mathrm{mmol} / \mathrm{L}$, 最低检测限 位 $10 \mu \mathrm{mol} / \mathrm{L}$ 。该传感器具有灵敏度高、抗干扰能力强以及稳定性好等特点。

关 键 词: 微球; 三元复合材料; 葡萄糖; 电化学传感

中图分类号: TQ174 文献标识码: A

\section{In Situ Preparation and Glucose Sensing Property of Ternary $\mathrm{NiO} / \mathrm{Ni} / \mathrm{C}$ Microspheres}

\author{
QIN Dong-Yu ${ }^{1,2}$, HE Xiao-Long ${ }^{1}$, NIE Qiu-Lin ${ }^{1}$, YIN Hao-Yong ${ }^{1}$, YUAN Qiu-Li ${ }^{2}$
}

(1. School of Material and Environmental Engineering, Hangzhou Dianzi University, Hangzhou 310018, China; 2. School of Science, Hangzhou Dianzi University, Hangzhou 310018, China)

\begin{abstract}
Ternary $\mathrm{NiO} / \mathrm{Ni} / \mathrm{C}$ microspheres was obtained by calcination of the precursor $\mathrm{Ni}(\mathrm{OH})_{2} / \mathrm{C}$ in pure nitrogen. The precursor was prepared via an one-pot hydrothermal synthesis technology using glucose, nickel chloride and urea. Scanning electron microscope (SEM), Transmission electron microscopy (TEM), X-ray diffraction (XRD), X-ray energy dispersive (EDS), and Raman were used to characterize the morphology and structure of the sample. Results show that the $\mathrm{NiO} / \mathrm{Ni} / \mathrm{C}$ microspheres have a coral-like structure with diameter of about $1.7 \mu \mathrm{m}$, and the crystalline phase of $\mathrm{Ni}$ and $\mathrm{NiO}$ are cubic. Electrochemical behavior and sensing property of ternary $\mathrm{NiO} / \mathrm{Ni} / \mathrm{C}$ microspheres composites were studied via cyclic voltammetry and amperometric method. When the $\mathrm{Ni} / \mathrm{NiO}$ molar ratio is 0.19 , the ternary $\mathrm{NiO} / \mathrm{Ni} / \mathrm{C}$ microspheres have excellent glucose sensor performance with a sensitivity of $241.09 \mu \mathrm{A} \cdot \mathrm{mmol} /\left(\mathrm{L} \cdot \mathrm{cm}^{2}\right)$ in a range of $10-5.05 \mathrm{mmol} / \mathrm{L}$, and a detecting limit of $10 \mu \mathrm{mol} / \mathrm{L}$. Furthermore, the sensor has good stability and anti-interference ability.
\end{abstract}

Key words: microspheres; ternary composites; glucose; electrochemical sensor

在临床医学上，糖尿病严重危害人类健康，其 诊断和治疗一直是医学界的一个重大难题。通过对
糖尿病患者血糖含量的准确检测，可以有效地对糖 尿病进行监测和治疗，因此葡萄糖的定量分析在临 
床医学上具有十分重要的意义 ${ }^{[1]}$ 。目前, 葡萄糖含量 分析检测方法有很多种, 如色谱法、光谱法和电化 学方法, 其中电化学方法以其具有灵敏度高、检出 限低、操作简便等优点得到广泛应用 ${ }^{[2-4]}$ 。电化学葡 萄糖传感器又可分为酶葡萄糖电化学传感器和无酶 葡萄糖电化学传感器, 其中酶葡萄糖电化学传感器 由于在电极构造、储存和使用过程中酶容易发生变 性, 并受外界环境的影响存在稳定性差、抗干扰能 力弱等问题 ${ }^{[5]}$; 而无酶葡萄糖电化学传感器不受酶 易变性失活的影响, 使用寿命长、制备成本低, 具有 稳定性好、易于重现、不易受环境影响等特点, 因 而受到越来越多的关注 ${ }^{[6]}$ 。

基于过渡金属氧化物尤其是 $\mathrm{NiO}$ 的非酶葡萄糖 电传感器, 因其价位低、活性高及在低电位处可促 进电子转移等特点, 作为电化学催化剂的各种材料 或基板已成为研究的热点 ${ }^{[7]}$ 。近年来 $\mathrm{NiO}$ 与其它材 料进行复合产生协同效应并提高葡萄糖电化学传感 性能的研究引起了人们广泛关注 ${ }^{[8]}$, 如纳米核壳结 构 $\mathrm{Ni} / \mathrm{NiO}^{[9]} 、 \mathrm{NiO} @ \mathrm{Ni}$ 同轴纳米线 ${ }^{[10]}$ 和 $\mathrm{NiO}$ 与石墨 烯复合材料 ${ }^{[11]}$ 等表现出更优化的葡萄糖电化学传 感性能。但是, 在纳米尺度上开展具有优异综合性 能的葡萄糖传感材料的设计、应用和机理探索, 依 然是一个十分重要且具有挑战性的课题。

本工作利用碳材料的强还原特性, 对过渡金属 氧化物 $\mathrm{NiO}$ 进行还原, 使金属导体、金属氧化物半 导体以及非金属碳三种完全不同材料进行原位复合, 能够形成界面结合力强、热力学稳定的 $\mathrm{NiO} / \mathrm{Ni} / \mathrm{C}$ 微 球三元复合纳米材料, 并将其应用于无酶葡萄糖传 感器中, 获得了优异的葡萄糖传感性能。

\section{1 实验方法}

\section{1 试剂}

葡萄糖(Glucose)、六水合氯化镍 $\left(\mathrm{NiCl}_{2} \cdot 6 \mathrm{H}_{2} \mathrm{O}\right)$ 、 尿素(Urea)、菜酚(Nafion)、无水乙醇(Ethanol absolute)、氢氧化钠 $(\mathrm{NaOH})$ 、颟氨酸 (L-valine)、脯氨 酸(L-proline)、赖氨酸(L-lysine)、氯化镍 $(\mathrm{NaCl})$, 所 用试剂均购于阿拉丁试剂(上海)有限公司, 且均为 分析纯，溶液用去离子水配置。

\section{2 实验仪器}

使用 CHI630D 电化学分析工作站的三电极体 系进行电化学测试。采用 S-4700 扫描电子显微镜、 JEOL 200CX 高分辨透射电镜、DX-2600 X 射线衍 射仪和 Renishaw 2000 型激光 Raman 谱仪等观察和 分析样品形貌、成分及晶体结构。

\section{$1.3 \mathrm{NiO} / \mathrm{Ni} / \mathrm{C}$ 微球的制备}

\subsection{1 $\mathrm{Ni}(\mathrm{OH})_{2} / \mathrm{C}$ 的合成}

将 $3.9 \mathrm{~g}$ 葡萄糖和 $1.18 \mathrm{~g} \mathrm{NiCl} \cdot 6 \mathrm{H}_{2} \mathrm{O}$ 溶于 $100 \mathrm{~mL}$ 去离子水中, 再加入 $3 \mathrm{~g}$ 尿素搅拌至澄清。 将溶液移至反应釜中, $180^{\circ} \mathrm{C}$ 下水热反应 $8 \mathrm{~h}$ 。待反应 釜冷却至室温后, 取自然底层沉淀经离心洗涤干燥, 获得前驱体 $\mathrm{Ni}(\mathrm{OH})_{2} / \mathrm{C}$ 。

\subsection{2 $\mathrm{NiO} / \mathrm{Ni} / \mathrm{C}$ 微球的合成}

将前驱体 $\mathrm{Ni}(\mathrm{OH})_{2} / \mathrm{C}$ 放入程序控制管式炉内, 先通入高纯度氮气除氧 $0.5 \mathrm{~h}$ 后, 然后在氮气氛围按 $7^{\circ} \mathrm{C} / \mathrm{min}$ 程序升温至 $400^{\circ} \mathrm{C}$ 并分别保温 $0.5 \mathrm{~h} 、 1 \mathrm{~h}$ 和 $2 \mathrm{~h}$, 冷却后获得的 $\mathrm{NiO} / \mathrm{Ni} / \mathrm{C}$ 微球依次表示为 $\mathrm{NiO} / \mathrm{Ni} / \mathrm{C}-1 、 \mathrm{NiO} / \mathrm{Ni} / \mathrm{C}-2 、 \mathrm{NiO} / \mathrm{Ni} / \mathrm{C}-3$ 。 $\mathrm{NiO} / \mathrm{C}$ 参照 样品按文献[12]中的方法制备。

\section{2 结果与讨论}

\section{$2.1 \mathrm{NiO} / \mathrm{Ni} / \mathrm{C}$ 微球的形貌与结构}

图 1 是不同样品的 XRD 图谱, 如图所示, 在 $2 \theta=37.15^{\circ} 、 43.20^{\circ} 、 62.78^{\circ} 、 75.23^{\circ} 、 79.28^{\circ}$ 处衍射峰 分别与 $\mathrm{NiO}$ 的(111)、(200)、(220)、(311)、(222) 晶 面相对应, 五个衍射峰均为立方相 $\mathrm{NiO}$ 衍射峰。在 $2 \theta=44.39^{\circ} 、 51.74^{\circ}$ 处出现明显的衍射峰, 对应面心立 方 $\mathrm{Ni}(111)$ 和(200)晶面 ${ }^{[13]}$, 这表明复合材料的氢氧 化镍前驱体受热分解后生成氧化镍，在高温氮气氛 围中由于碳材料存在一定还原性，部分氧化镍被原 位还原成了单质镍 ${ }^{[14-15]}$ 。从图 1 可以看出, 随着煅 烧时间的延长, $2 \theta=44.39^{\circ}(\mathrm{Ni}(111))$ 处的衍射峰逐渐 增强, 并且最后在 $2 \theta=51.74^{\circ}$ 也出现了 $\mathrm{Ni}(200)$ 晶面 的衍射峰, 表明样品中 $\mathrm{Ni}$ 含量随着炦烧时间的延长 而增加。根据 $\mathrm{Ni}(111)$ 和相邻 $\mathrm{NiO}(200)$ 衍射峰的积分

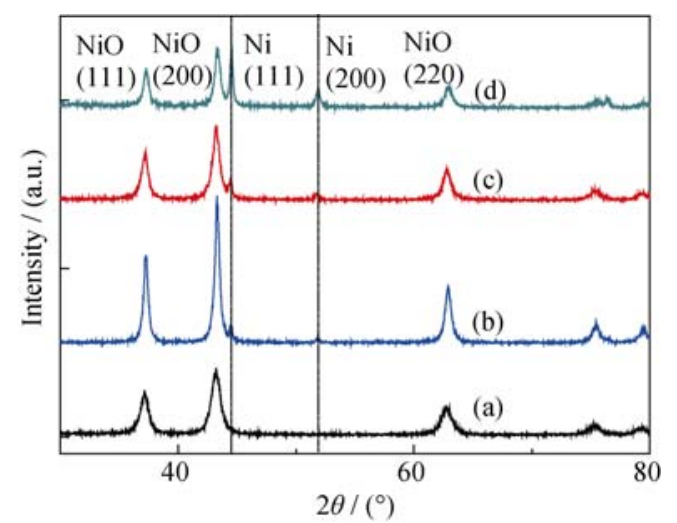

图 $1 \mathrm{NiO} / \mathrm{C}(\mathrm{a}) 、 \mathrm{NiO} / \mathrm{Ni} / \mathrm{C}-1(\mathrm{~b}) 、 \mathrm{NiO} / \mathrm{Ni} / \mathrm{C}-2(\mathrm{c})$ 和 $\mathrm{NiO} / \mathrm{Ni} / \mathrm{C}-3$ (d)的 XRD 图谱

Fig. 1 XRD patterns of $\mathrm{NiO} / \mathrm{C}(\mathrm{a}), \mathrm{NiO} / \mathrm{Ni} / \mathrm{C}-1(\mathrm{~b}), \mathrm{NiO} / \mathrm{Ni} /$ $\mathrm{C}-2$ (c), and $\mathrm{NiO} / \mathrm{Ni} / \mathrm{C}-3$ (d) 
面积比值可以计算出不同样品中 $\mathrm{Ni} / \mathrm{NiO}$ 的摩尔比 约为 $0(\mathrm{NiO}) 、 0.12(\mathrm{NiO} / \mathrm{Ni} / \mathrm{C}-1) 、 0.19(\mathrm{NiO} / \mathrm{Ni} / \mathrm{C}-2)$ 和 $0.56(\mathrm{NiO} / \mathrm{Ni} / \mathrm{C}-3)$ 。

图 2 为 $\mathrm{NiO} / \mathrm{Ni} / \mathrm{C}-2$ 样品的 Raman 光谱图, 在 1141 和 $1590 \mathrm{~cm}^{-1}$ 处可观察到两个尖锐谱峰, 分别对应 $\mathrm{NiO}$ 和 $\mathrm{C}$ 的特征峰, 结合 XRD 分析结果, 表明 $\mathrm{NiO} / \mathrm{Ni} / \mathrm{C}-2$ 样品含有 $\mathrm{NiO} 、 \mathrm{Ni}$ 和 $\mathrm{C}$ 成分。图 3(a) (d) 是不同样品 的 SEM 照片, 可以看出样品形貌均呈微球结构, 微球 直径范围在 1.1 2.5 $\mu \mathrm{m}$ 。图 3(e) 是 $\mathrm{NiO} / \mathrm{Ni} / \mathrm{C}-2$ 样品的 高倍 SEM 照片, 可以看出微球呈珊瑚花状, 由许多纳 米片构成, 纳米片厚度约为 $36 \mathrm{~nm}$ 。图 3(f) 为 $\mathrm{NiO} / \mathrm{Ni} / \mathrm{C}-2$ 样品的 EDS 能谱图, 显示其化学元素组成 有镍、氧和碳元素, 其中铂元素的信号来源于样品表 面导电镀层铂。

图 4(a) 是 $\mathrm{NiO} / \mathrm{Ni} / \mathrm{C}-2$ 样品微球的透射电镜照片, 可以看出, 构成微球的纳米片由大量纳米颗粒构 成。由图 4(b)的 HRTEM 照片可以进一步看到 $\mathrm{NiO} / \mathrm{Ni} / \mathrm{C}-2$ 微球的两个不同晶面间距为 $0.207 \mathrm{~nm}$ 和 $0.246 \mathrm{~nm}$, 分别对应 $\mathrm{Ni}$ 的(101)晶面和 $\mathrm{NiO}$ 的(111) 晶面 ${ }^{[16-17]}$, 该结果与 XRD 分析结果相一致。

\section{$2.2 \mathrm{NiO} / \mathrm{Ni} / \mathrm{C}$ 微球的电化学行为}

图 5 为不同保温时间获得的 $\mathrm{NiO} / \mathrm{Ni} / \mathrm{C}$ 微球在扫 描速率为 $50 \mathrm{mV} / \mathrm{s}$, 葡萄糖浓度为 $1 \mathrm{mmol} / \mathrm{L}$ 的 $\mathrm{NaOH}$ 溶液 $(0.1 \mathrm{~mol} / \mathrm{L})$ 中测得的循环伏安曲线。图 5 显示, 样品 $\mathrm{NiO} / \mathrm{Ni} / \mathrm{C} /-3$ 未出现明显的氧化还原峰, 另外三个样品均出现明显的氧化还原峰, 其中 $\mathrm{NiO} / \mathrm{Ni} / \mathrm{C}-2$ 的氧化还原峰值最高。

不同保温时间获得 $\mathrm{NiO} / \mathrm{Ni} / \mathrm{C}$ 微球的镍含量不 同。少量金属单质 $\mathrm{Ni}$ 的复合可以增强 $\mathrm{NiO} / \mathrm{Ni} / \mathrm{C}$ 复 合材料的导电性, 从而提高电极的电荷传输速度, 并有助于电化学反应动力学 ${ }^{[18]}$ 。过量 $\mathrm{Ni}$ 的复合会 降低电极材料中活性物质 $\mathrm{NiO}$ 的含量, 因此可能导

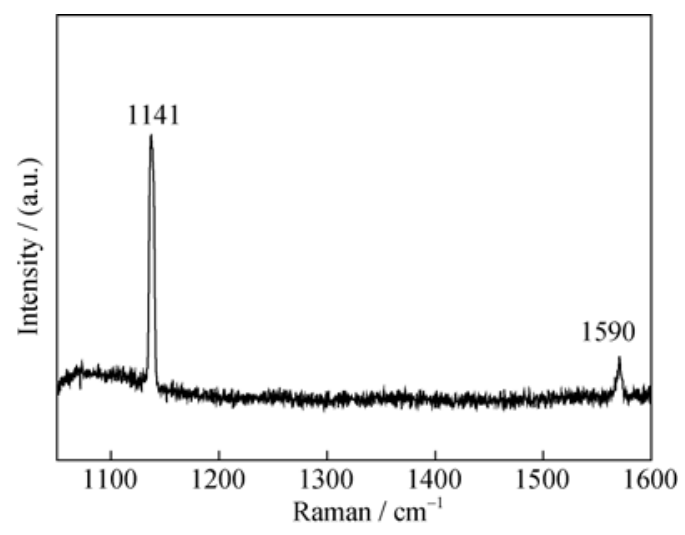

图 $2 \mathrm{NiO} / \mathrm{Ni} / \mathrm{C}-2$ 样品的 Raman 光谱图

Fig. 2 Raman spectrum of $\mathrm{NiO} / \mathrm{Ni} / \mathrm{C}-2$ sample
致电化学性能的降低。很明显, 当 $\mathrm{Ni} / \mathrm{NiO}$ 摩尔比为 0.19 , 形成的 $\mathrm{NiO} / \mathrm{Ni} / \mathrm{C}$ 微球中 $\mathrm{Ni}$ 含量适中, 因而 $\mathrm{NiO} / \mathrm{Ni} / \mathrm{C}-2$ 的氧化还原峰值最高, 具有最好的电化 学催化活性。

图 6 为不同保温时间获得的 $\mathrm{NiO} / \mathrm{Ni} / \mathrm{C}$ 微球的电 化学阻抗谱, 从图中看出, $\mathrm{NiO} / \mathrm{Ni} / \mathrm{C}-2$ 微球样品的 阻抗谱半圆半径最小, 表明 $\mathrm{NiO} / \mathrm{Ni} / \mathrm{C}-2$ 微球样品具 有更好的导电性和更低的扩散和电荷传输阻抗，以 及优良的电化学传感活性。阻抗试验和循环伏安的 结果也相吻合, 都显示出 $\mathrm{NiO} / \mathrm{Ni} / \mathrm{C}-2$ 微球具有良好 的电化学性能。

图 7 为 $\mathrm{NiO} / \mathrm{Ni} / \mathrm{C}-2$ 修饰电极在不同葡萄糖浓度 的氢氧化钠溶液中 $(0.1 \mathrm{~mol} / \mathrm{L})$ 的循环伏安图, 可以 看出, 氧化峰电流随葡萄糖浓度增加而增大, 这说 明在葡萄糖和电极之间发生了直接的电子转移。

\section{$2.3 \mathrm{NiO} / \mathrm{Ni} / \mathrm{C}$ 微球的葡萄糖传感性能}

图 8 给出了不同构建的传感器对葡萄糖的电流 响应曲线, 在 $0.5 \mathrm{~V}$ 电压下, 连续向氢氧化钠溶液 $(0.1 \mathrm{~mol} / \mathrm{L})$ 中加入不同浓度的葡萄糖溶液。从图 8 可看出传感器均出现了安培响应。在相同的葡萄糖 浓度下, $\mathrm{NiO} / \mathrm{Ni} / \mathrm{C}-2$ 修饰电极对葡萄糖的催化表现 与其他修饰电极相比更优异, 这与循环伏安测定的 结果一致。

图 8 插图为 $\mathrm{NiO} / \mathrm{Ni} / \mathrm{C}-2$ 修饰电极在氢氧化钠溶 液 $(0.1 \mathrm{~mol} / \mathrm{L})$ 中连续滴加不同浓度葡萄糖溶液, 各葡 萄糖浓度与响应电流的线性关系，可以看到，响应 电流值随葡萄糖浓度的增加而增大。当电流差值达 到检测限时逐渐降低, 电流曲线趋于平缓。图中拟合 直线为: $I=0.01202+1.70331 \times 10^{-5} C$, 对应葡萄糖浓 度范围是 $10 \sim 5.05 \mathrm{mmol} / \mathrm{L}\left(R^{2}=0.99\right)$ 。 $\mathrm{NiO} / \mathrm{Ni} / \mathrm{C}-2$ 所 修饰电极的灵敏度为 $241.09 \mu \mathrm{A} \cdot \mathrm{mmol} /\left(\mathrm{L} \cdot \mathrm{cm}^{2}\right)$ 。在信 噪比 $S / N=3$ 时, 最低检测限约为 $10 \mu \mathrm{mol} / \mathrm{L}$ 。

\section{4 传感器的抗干扰性、稳定性和回收率}

高选择性是评估非酶葡萄糖电化学传感器的另 一个重要因素。血糖中与葡萄糖共同存在的还有如 抗坏血酸、尿素、氨基酸等活性物质, 在实际检测中 这些活性物质常常会对传感器检测造成干扰。因此 研究干扰物质对 $\mathrm{NiO} / \mathrm{Ni} / \mathrm{C}-2$ 葡萄糖传感器的影响十 分关键。图 9 展示了在 $0.5 \mathrm{~V}$ 电压下分别向 $0.1 \mathrm{~mol} / \mathrm{L}$ $\mathrm{NaOH}$ 溶液中加入 $0.5 \mathrm{mmol} / \mathrm{L}$ 葡萄糖、 $0.05 \mathrm{mmol} / \mathrm{L}$ L-烦氨酸、 $0.05 \mathrm{mmol} / \mathrm{L}$ 尿素、 $0.05 \mathrm{mmol} / \mathrm{L}$ L-脯氨酸、 $0.05 \mathrm{mmol} / \mathrm{L}$ 氯化钠和 $0.05 \mathrm{mmol} / \mathrm{L} \mathrm{L}$-赖氨酸的电流 响应情况。按图 9 显示推算, 若在实际血糖检测中, 整个葡萄糖传感器受尿素、氯化钠、氨基酸的影响 几乎可以忽略。 

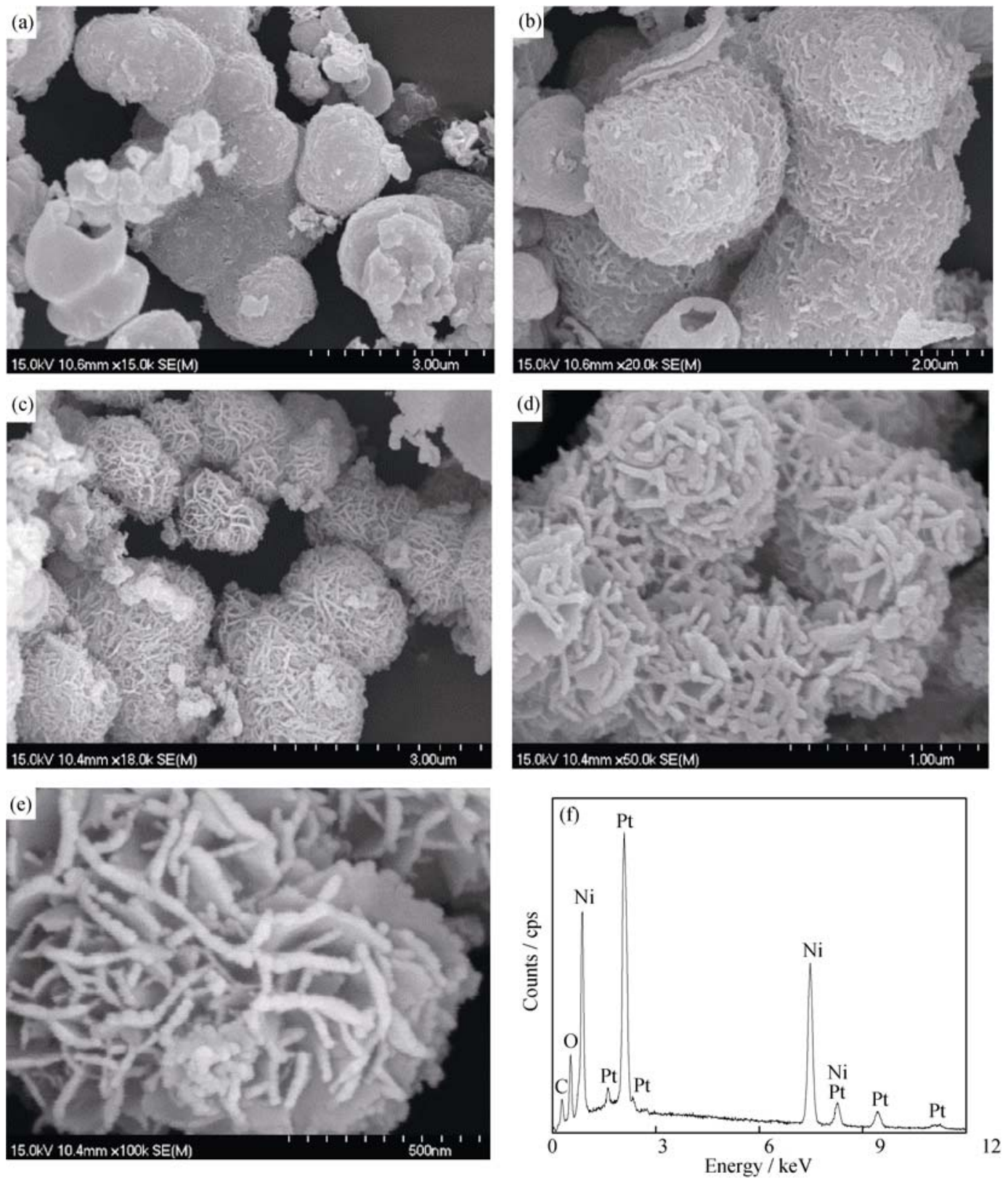

图 3 样品 $\mathrm{NiO} / \mathrm{C}(\mathrm{a}) 、 \mathrm{NiO} / \mathrm{Ni} / \mathrm{C}-1$ (b)、 $\mathrm{NiO} / \mathrm{Ni} / \mathrm{C}-2$ (c) 和 $\mathrm{NiO} / \mathrm{Ni} / \mathrm{C}-3(\mathrm{~d})$ 的 $\mathrm{SEM}$ 照片, $\mathrm{NiO} / \mathrm{Ni} / \mathrm{C}-2$ 的高倍 $\mathrm{SEM}$ 照片(e) 以及 $\mathrm{NiO} / \mathrm{Ni} / \mathrm{C}-2$ 的 EDS 能谱图(f)

Fig. 3 SEM images of $\mathrm{NiO} / \mathrm{C}(\mathrm{a}), \mathrm{NiO} / \mathrm{Ni} / \mathrm{C}-1$ (b), $\mathrm{NiO} / \mathrm{Ni} / \mathrm{C}-2$ (c), and $\mathrm{NiO} / \mathrm{Ni} / \mathrm{C}-3$ (d), as well as enlarged image of NiO/Ni/C-2 (e), and its EDS pattern of $\mathrm{NiO} / \mathrm{Ni} / \mathrm{C}-2$ (f)
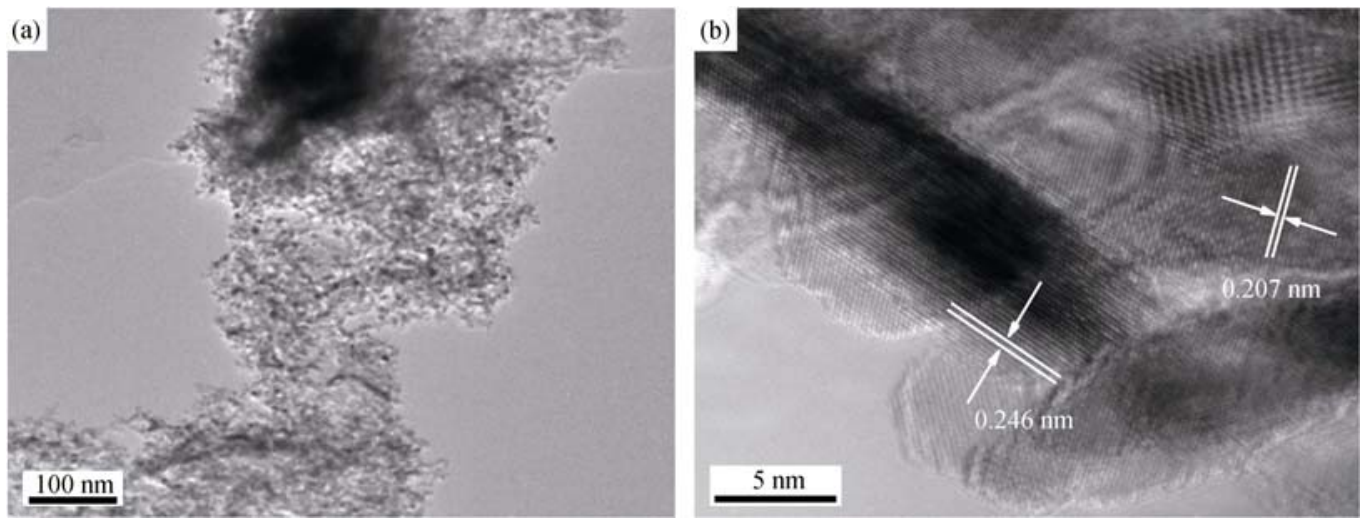

图 $4 \mathrm{NiO} / \mathrm{Ni} / \mathrm{C}-2$ 样品的 TEM 照片和 HRTEM 照片

Fig. 4 TEM (a) and HRTEM(b) images of $\mathrm{NiO} / \mathrm{Ni} / \mathrm{C}-2$ 


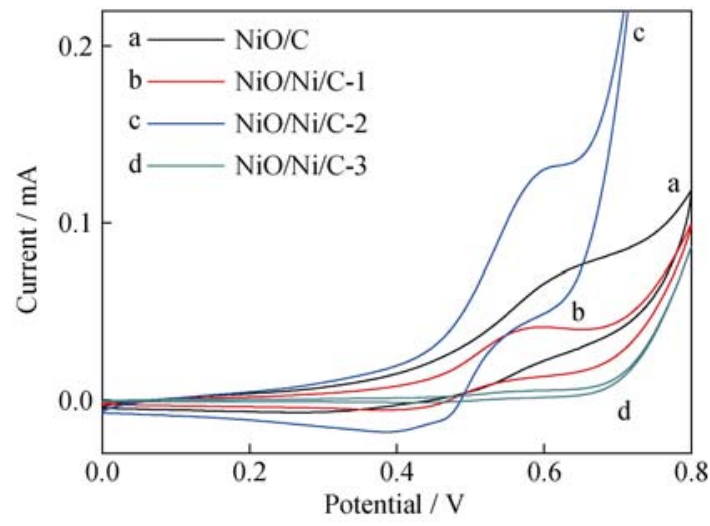

图 5 不同样品在 $1 \mathrm{mmol} / \mathrm{L}$ 葡萄糖溶液中的 $\mathrm{CV}$ 曲线

Fig. $5 \mathrm{CV}$ curves of different samples at $1 \mathrm{mmol} / \mathrm{L}$ glucose solution

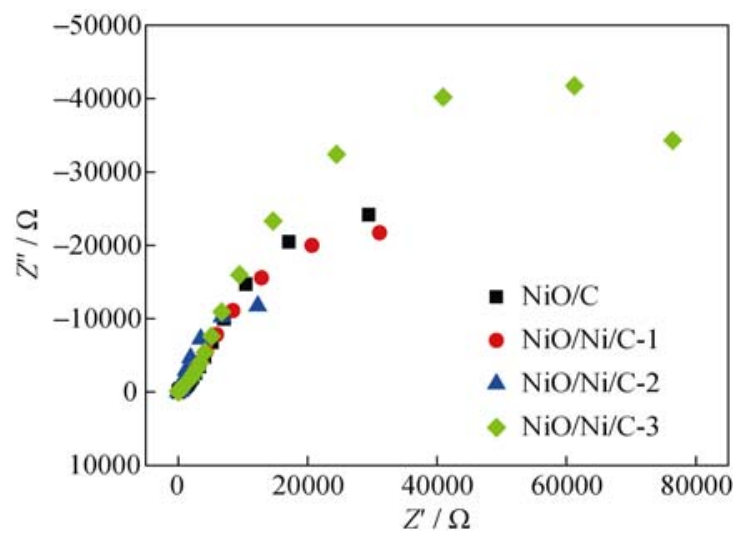

图 6 不同样品的电化学阻抗谱

Fig. 6 EIS spectra of different samples

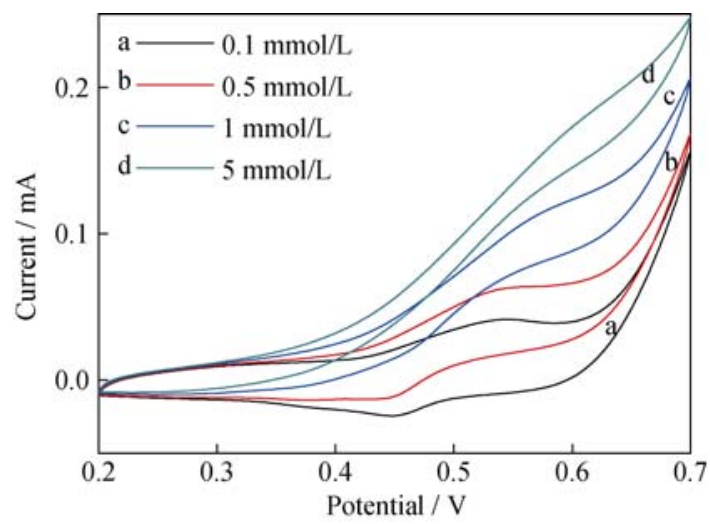

图 $7 \mathrm{NiO} / \mathrm{Ni} / \mathrm{C}-2 / \mathrm{GCE}$ 在不同浓度葡萄糖溶液中以扫描 速率为 $50 \mathrm{mV} / \mathrm{s}$ 的 $\mathrm{CV}$ 曲线

Fig. $7 \mathrm{CV}$ of $\mathrm{NiO} / \mathrm{Ni} / \mathrm{C}-2 / \mathrm{GCE}$ in glucose solution with different concentrations at scan rate of $50 \mathrm{mV} / \mathrm{s}$

采用标准加入法在 $0.58 \mathrm{~V}$ 电压、 $0.1 \mathrm{~mol} / \mathrm{L}$ $\mathrm{NaOH}$ 溶液中对 4 份 $0.5 \mathrm{~mol} / \mathrm{L}$ 葡萄糖溶液样品进 行了加标回收测定, 结果见表 1 。从表 1 可以看出, 四份葡萄糖样品的加标回收率在 $95 \%$ 109.5\%之 间，表明制备的传感器可用于实际葡萄糖的测定。

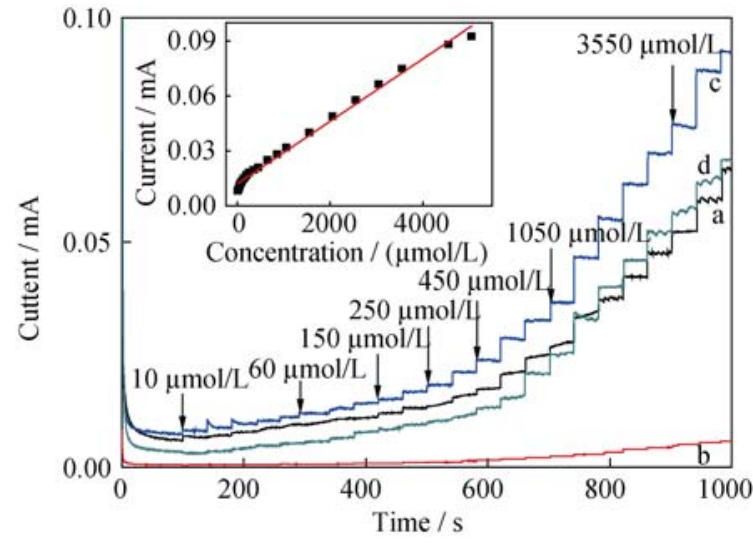

图 8 样品 $\mathrm{NiO} / \mathrm{C}(\mathrm{a}) 、 \mathrm{NiO} / \mathrm{Ni} / \mathrm{C}-1(\mathrm{~b}) 、 \mathrm{NiO} / \mathrm{Ni} / \mathrm{C}-2(\mathrm{c})$ 和 $\mathrm{NiO} / \mathrm{Ni} / \mathrm{C}-3(\mathrm{~d})$ 葡萄糖传感器的 $i-t$ 曲线

Fig. $8 i$ - $t$ curves of $\mathrm{NiO} / \mathrm{C}$ (a) , NiO/Ni/C-1 (b), NiO/Ni/C-2 (c) and $\mathrm{NiO} / \mathrm{Ni} / \mathrm{C}-3$ (d) glucose sensors

Inset shows linear calibration of $\mathrm{NiO} / \mathrm{Ni} / \mathrm{C}-2$ sensors between current response and glucose concentration

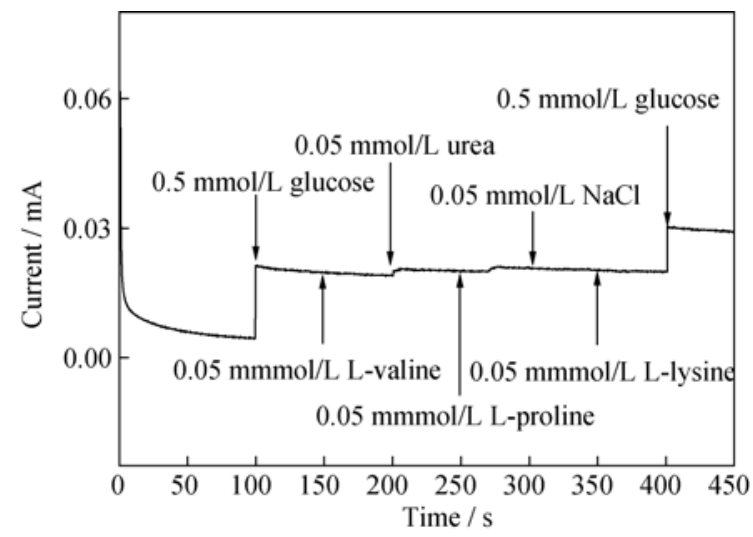

图 $9 \mathrm{NiO} / \mathrm{Ni} / \mathrm{C}-2$ 葡萄糖传感器的干扰测试

Fig. 9 Interference test of $\mathrm{NiO} / \mathrm{Ni} / \mathrm{C}-2$ glcose sensor

表 1 葡萄糖回收率的测定值

Table 1 Determination of glucose recovery

\begin{tabular}{ccc}
\hline Spiked $/\left(\mathrm{mmol} \cdot \mathrm{L}^{-1}\right)$ & Found $/\left(\mathrm{mmol} \cdot \mathrm{L}^{-1}\right)$ & Recovery $/ \%$ \\
\hline 0.21 & 0.20 & 95.0 \\
0.41 & 0.42 & 104.8 \\
0.61 & 0.63 & 109.5 \\
0.81 & 0.82 & 104.7 \\
\hline
\end{tabular}

将测试过的 $\mathrm{NiO} / \mathrm{Ni} / \mathrm{C}-2$ 修饰电极放置 $4 \mathrm{~d}$ 后再 测试其葡萄糖传感性能, 所得的电流响应几乎无差 异, 说明 $\mathrm{NiO} / \mathrm{Ni} / \mathrm{C}-2$ 微球构造的葡萄糖传感器反应 过程稳定, 传感性能良好, 具有较出色的抗干扰性。

\section{3 结论}

通过一步水热法制备了 $\mathrm{NiO} / \mathrm{Ni} / \mathrm{C}$ 三元复合微 球材料, 当 $\mathrm{Ni} / \mathrm{NiO}$ 摩尔比为 0.19 时, 形成的 $\mathrm{NiO} / \mathrm{Ni} / \mathrm{C}-2$ 三元复合微球显示了对葡萄糖较高的电 
催化氧化活性: 其灵敏度为 $241.9 \mu \mathrm{A} \cdot \mathrm{mmol} /\left(\mathrm{L} \cdot \mathrm{cm}^{2}\right)$, 线性响应范围是 $10 \mu \mathrm{mol} / \mathrm{L} \sim 5.05 \mathrm{mmol} / \mathrm{L}$, 最低检测 限为 $10 \mu \mathrm{mol} / \mathrm{L}$ 。同时, 由其构建的葡萄糖传感器具 有良好的抗干扰性和稳定性。

\section{参考文献:}

[1] WANG H C, LEE A R. Recent developments in blood glucose sensors. Journal of Food and Drug Analysis, 2015, 23: 191-200.

[2] GALANT S L, KAUFMAN R C, WILSON J D. Glucose: Detection and analysis. Food Chemistry, 2015, 188: 149-160.

[3] LU XIANBO, WEN ZHENHAI, LI JINGHONG. Hydroxylcontaining antimony oxide bromide nanorods combined with chitosan for biosensors. Biomaterials, 2006, 27(27): 57405747.

[4] YAN L, YANG L, HONGBIN F, et al. Layer-by-layer assembly of chemical reduced graphene and carbon nanotubes for sensitive electrochemical immunoassay. Biosensors \& Bioelectronics, 2012, 35(1): 63-68.

[5] ZHAO DE-RUI, ZHAI YING-JIAO, LI JIN-HUA, et al. Preparation and properties of glucose biosensor based on flower-like $\mathrm{MoS}_{2}$ micrometer material. Journal of Inorganic Materials, 2016, 31(2): 153-158.

[6] SENTHAMIZHAN A, BALUSAMY B, UYAR T. Glucose sensors based on electrospun nanofibers: a review. Analytical \& Bioanalytical Chemistry, 2016, 408(5): 1285-1306.

[7] BAGHAYERI M, AMIRI A, FARHADI S. Development of non-enzymatic glucose sensor based on efficient loading $\mathrm{Ag}$ nanoparticles on functionalized carbon nanotubes. Sensors and Actuators B-Chemical, 2016, 225: 354-362.

[8] YAO M M, HU Z H, XU Z J, et al. High-performance electrode materials of hierarchical mesoporous nickel oxide ultrathin nanosheets derived from self-assembled scroll-like a-nickel hydroxide. Journal of Power Sources, 2015, 273: 914-922.

[9] ZHANG C, QIAN L H, ZHANG K, et al. Hierarchical porous $\mathrm{Ni} / \mathrm{NiO}$ core-shells with superior conductivity for electro- chemical pseudocapacitors and glucose sensors. J. Mater. Chem. A, 2015, 3: 10519-10525.

[10] JAMAL M, HASAN M, SCHMIDT M, et al. Shell@core coaxial NiO@Ni nanowire arrays as high performance enzymeless glucose sensor. Journal of the Electrochemical Society, 2013, 160: B207-B212.

[11] LI S J, XIA N, LV X L. A facile one-step electrochemical synthesis of graphene/ $\mathrm{NiO}$ nanocomposites as efficient electrocatalyst for glucose and methanol. Sensors and Actuators B: Chemical, 2014, 190: 809-817.

[12] CUI ZHEN-ZHEN, YIN HAO-YONG, ZHAO HONG-TING, et $a l$. Preparation and glucose sensing property of core-shelled nikel oxide/carbon microspheres. Journal of Inorganic Materials, 2015, 3: 305-310.

[13] LIU XIANG GUO, JIN CHUANGUI, FENG CHAO, et al. $\mathrm{NiO} / \mathrm{C}$ nano-capsules with onion-like carbon shell as anode material for lithium ion batteries. Carbon, 2013, 60: 215-220.

[14] LUO CHENG HAO, LU WEI LI, LI YU, et al. Preparation of $\mathrm{C} / \mathrm{Ni}-\mathrm{NiO}$ composite nanofibers for anode materials in lithium-ion batteries, Applied Physics A, 2013, 113: 683-692.

[15] LUO LI QIANG, LI FANG, ZHU LI MEI, et al. Non-enzymatic glucose sensor based on nickel (II) oxide/ordered mesoporous carbon modified glassy carbon electrode, Colloids and Surfaces B: Bio-interfaces, 2013, 102: 307-311.

[16] GOKUL B, SARAVANAN P, VINOD V T P, et al. Synthesis of $\mathrm{Ni} / \mathrm{NiO}$ nanocomposites by hydrothermal-assisted polyol process and their magnetic properties as a function of annealing temperature. Powder Technology, 2015, 274: 98-104.

[17] FENG YY, ZHANG H J, LI W X, et al. Targeted synthesis of novel hierarchical sandwiched $\mathrm{NiO} / \mathrm{C}$ arrays as high-efficiency lithium ion batteries anode. Journal of Power Sources, 2016, 301: 78-86.

[18] CHEN M H, ZHANG J W, XIA X H, et al. Self-supported Ni decorated $\mathrm{NiO}$ nano-flake arrays as promising cathode materials of hybrid batteries. Materials Research Bulletin, 2016, 76: 113-117. 